Experimental and theoretical investigation on the misalignment tolerance of a micron-sized solid immersion lens

This article has been downloaded from IOPscience. Please scroll down to see the full text article.

2013 J. Opt. 15025706

(http://iopscience.iop.org/2040-8986/15/2/025706)

View the table of contents for this issue, or go to the journal homepage for more

Download details:

IP Address: 128.178.203.152

The article was downloaded on 11/01/2013 at 16:30

Please note that terms and conditions apply. 


\title{
Experimental and theoretical investigation on the misalignment tolerance of a micron-sized solid immersion lens
}

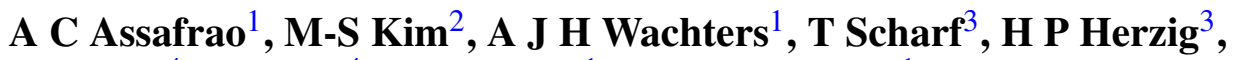

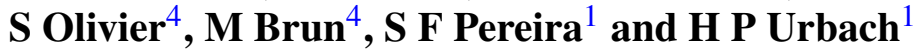 \\ ${ }^{1}$ Optics Research Group, Department of Imaging Science and Technology, Delft University of \\ Technology, Lorentzweg 1, 2628 CJ Delft, The Netherlands \\ 2 SUSS MicroOptics SA, Rue Jaquet-Droz 7, CH-2000 Neuchatel, Switzerland \\ ${ }^{3}$ Optics and Photonics Technology Laboratory, Ecole Polytechnique Federale de Lausanne (EPFL), \\ Rue A.-L. Breguet 2, Neuchatel, CH-2000, Switzerland \\ ${ }^{4}$ CEA-Leti, Minatec Campus, 17 rue des Martyrs, 38054 Grenoble Cedex 9, France
}

E-mail: a.dacostaassafrao@tudelft.nl

Received 10 September 2012, accepted for publication 3 December 2012

Published 3 January 2013

Online at stacks.iop.org/JOpt/15/025706

\begin{abstract}
We report an experimental and theoretical study on the alignment error tolerance of a $2 \mu \mathrm{m}$-size solid immersion lens (SIL) illuminated by different types of focused spots. Tightly confined focal spots are of great interest for improving the performance of many optical systems, so that a study on the alignment tolerance is of interest. In particular, it was found that micro-SILs can be largely misaligned with respect to the optical axis of an objective lens focusing light onto it and yet allow for a reasonably good immersed spot. In fact, a displacement of approximately $400 \mathrm{~nm}$, i.e. one fifth of the lens diameter, is tolerable. The measurements are compared with a rigorous finite element method model for a micro-SIL, showing an excellent agreement.
\end{abstract}

Keywords: micro-SIL, misalignment tolerance, solid immersion lens

(Some figures may appear in colour only in the online journal)

\section{Introduction}

The recent demand for miniaturized high-quality optical systems for strong light confinement has pushed forward the development of solid immersion lenses (SILs), down to the micrometre-scale [1-5]. These micro-solid immersion lenses $(\mu$-SIL) have a potential to be applied in many different fields, such as microscopy [6], lithography [7], optical data storage [8], Raman spectroscopy [9], and fluorescent imaging [10]. Contrary to the liquid immersion technique, the utilization of SIL is desirable for applications in which liquids may harm or contaminate the sample. Optical data storage and lithography are examples of such applications.
Moreover, there are many materials with high refractive index value that can be potentially used for fabrication of SILs. In order to maximize the focusing capabilities of the $\mu$-SIL, this tiny SILs should be placed at the focal plane of a high numerical aperture (NA) objective lens, resulting in an immersed spot whose dimensions are reduced by a factor equal to the refractive index of the material of the SIL. Optical trapping is a particular interesting application for the $\mu$-SIL, where a central dark zone of an immersed doughnut-like focused spot is used to trap and manipulate tiny objects that are repelled and pushed away from the regions of maximum intensity [11]. To achieve such an illumination spot, the state of polarization of a laser beam incident on the 


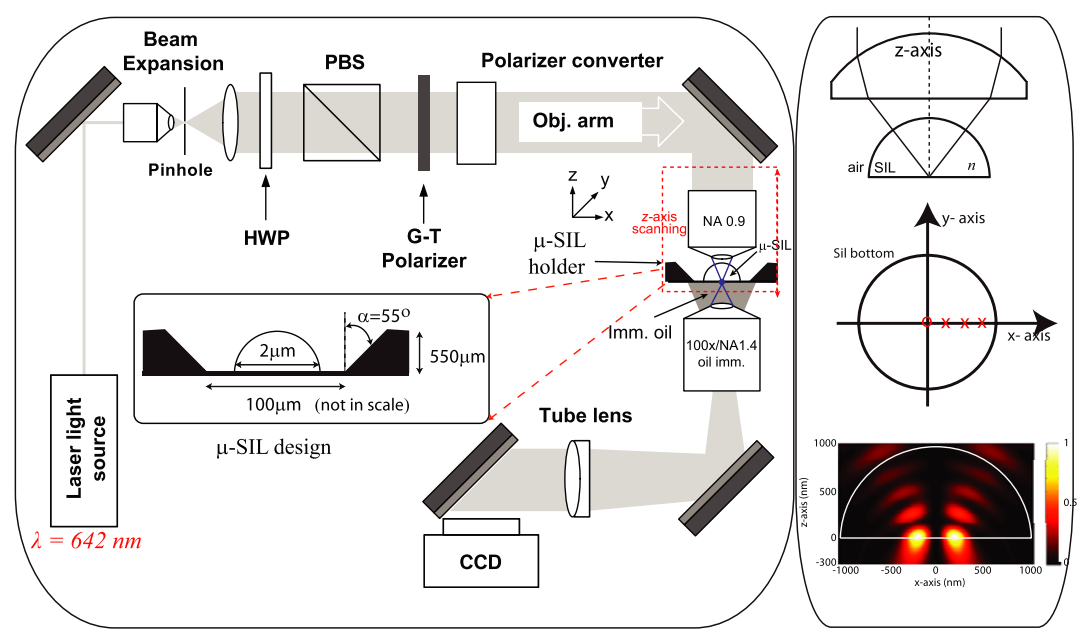

Figure 1. Left box: schematic of the experimental setup of the HRIM. In the object arm, an azimuthally polarized laser beam is normally incident on the focusing lens. The $\mu$-SIL and the NA $=0.9$ focusing lens are inserted on the same sample stage. By scanning the $\mu$-SIL together with the focusing lens along the $z$-axis, 3D maps of the focused field are obtained. Misalignment is inflicted on the $\mu$-SIL prior to the measurements. Right box: top-working principle of a solid immersion lens. The $\mu$-SIL is perfectly positioned with its centre coincident with the $z$-axis. Centre-a misalignment is inflicted on the $\mu$-SIL by shifting its centre to positions along the $x$-direction (the focal plane of the objective lens remains unaltered). Bottom-computational domain of the 3D FEM model for the $\mu$-SIL. The $\mu$-SIL, described as a semi-hemispherical structure, is placed in the focal plane of an objective lens. The immersed focal spot, collected on the bottom of the $\mu$-SIL lens, will be used for comparisons with the measurements.

objective lens is set to azimuthal, so that a doughnut-shaped spot is generated on the focal plane [12]. Since the $\mu$-SIL is positioned on that plane, the final spot will have not only its dimensions reduced, but also its field intensity increased on the ring structure, as a result of the energy confinement. A spot with such features, i.e. a central dark region surrounded by a high intensity annular region, is highly desirable for efficiently trapping tiny particles, in particular photosensitive materials such as biological cells and neutral atoms [13], as well as for stimulated emission depletion (STED) microscopy techniques [14]. Another interesting type of illumination is achieved by adding an extra linear polarizer in the light path of the azimuthally polarized beam. This particular configuration leads to a decomposition of the azimuthal polarization, resulting in two half-lobes whose orientation depends upon the orientation of the polarizer. Such two-lobe spots can be applied in direct-write vortex beam lithography, which can produce well-separated line patterns of visible light. Similarly to the previous case, when this type of spot is immersed, its dimensions are also reduced, which theoretically would allow for writing even smaller line patterns $[15,16]$.

At a practical level, one point of great concern certainly regards the alignment sensitivity of such micro-sized SILs. In other words, considering that the $\mu$-SIL should be ideally centred on the optical axis (z-axis) of the high NA lens (see the top diagram in the right box of figure 1), what is the consequence for the immersed focused spot if any misalignment occurs? Or even, what is the maximum misalignment tolerance which does not compromise the integrity of the immersed spot? Answering these questions is of great relevance not only for optical trapping but also for any application that may profit from the $\mu$-SIL. In this work, we numerically and experimentally study the alignment tolerance of a high numerical aperture objective lens $(\mathrm{NA}=0.9)$ focusing different types of spots onto a $2 \mu \mathrm{m}$ diameter hemispherical $\mathrm{SiO}_{2} \mu$-SIL [17]. The measurements are conducted using a high-resolution interference microscope (HRIM), which has been proven to be a powerful tool for 3D optical characterizations of micro-, and nano-optical elements [18], whereas the simulations are rigorously executed in a three-dimensional (3D) finite element method (FEM) simulation tool [19-21]. The simulation model is finally extended to compute the misalignment tolerance of $\mu$-SIL having different diameters or higher refractive index.

\section{Experimental and theoretical procedures}

The HRIM setup is depicted in the left box of figure 1 . A single mode polarized laser diode (CrystaLaser, $642 \mathrm{~nm}$ : DL640-050-3) is redirected to the object arm of the microscope after being expanded and collimated by a spatial filtering technique. An objective lens of NA $=0.9$ is used to focus the normally incident and azimuthally polarized laser beam, generated by a radial/azimuthal polarization converter (ARCoptix S.A.). To realize the two half-lobes illumination, an extra linear polarizer is placed before the objective lens. The objective lens is mounted on a precision piezo-stage with a $z$-scan range of $500 \mu \mathrm{m}$ and a $x-y$ scan range of $100 \mu \mathrm{m}$ (MAD CITY LABS). The $\mu$-SIL is held by a $100 \mathrm{~nm}$ thick silicon nitride membrane in a silicon chip, which has a thickness of $550 \mu \mathrm{m}$. The $\mu$-SIL chip is placed horizontally such that the planar side of the SIL faces the collector lens. Furthermore, the $\mu$-SIL is positioned in the focal plane of the focusing objective, also on the $z$-axis piezo-stage. As can be seen, the measurements are conducted in transmission. The complete description of the 


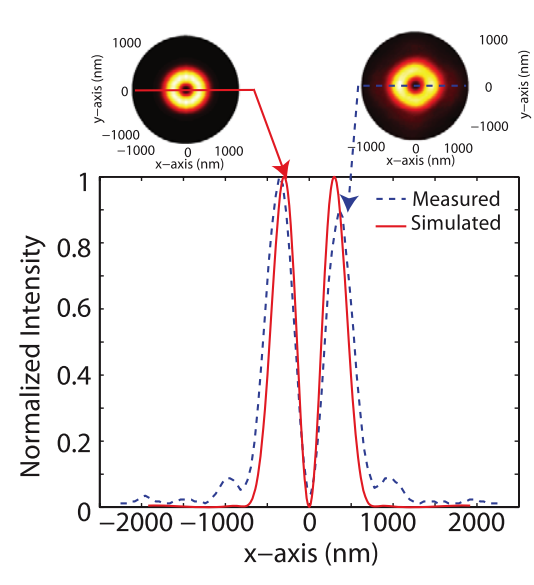

(a)

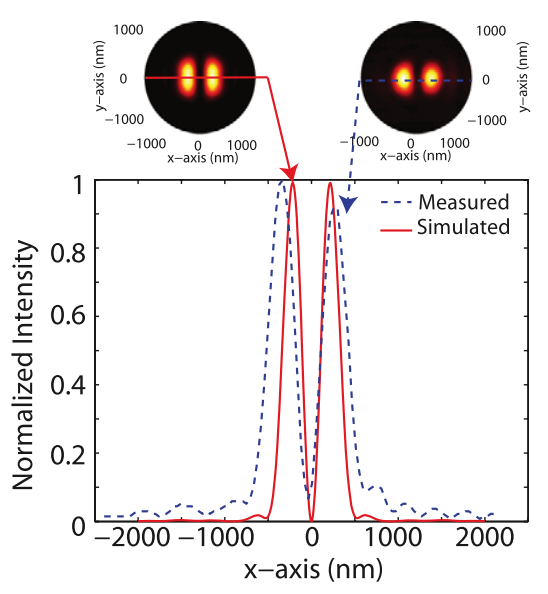

(b)

Figure 2. Measured and simulated intensity profiles of the on-axis immersed spots. (a) Doughnut-like immersed spot and (b) a two half-lobe immersed spot. The measured central dark region shows a good agreement with that of the simulation. All intensities are normalized.

HRIM experimental setup can be found in [18]. The SILs were fabricated on $200 \mathrm{~mm}$ silicon wafers by using standard microelectronic and microsystem tools. First, a hemispherical mould is formed by isotropic wet etching of silicon through a nanometric hole. Then a conformal thin layer of $100 \mathrm{~nm}$ silicon nitride $(\mathrm{SiN})$ is deposited on the substrate. This $\mathrm{SiN}$ layer will serve as the membrane holding the SIL at the end of the process. The hemispherical mould is then filled with silicon dioxide $\mathrm{SiO}_{2}$ material and a chemical-mechanical polishing step is performed to planarize the structure. Finally, the spherical surface of the SILs is released to the air by etching the substrate from the backside. The SILs are held by the $100 \mathrm{~nm}$ SiN membrane and can be used directly as an optical head to scan samples. Further details are reported in [17]. An important aspect of the current SIL design used in our experiments is the fact that the side wall angle of the $\mathrm{Si}$ substrate ultimately limits the maximum angle of the incoming focusing light. A schematic of the design of the SIL is also provided in figure 1. By simply applying geometrical optics, one can conclude that the size of the beam focused by a NA $=0.9$ at a distance of $550 \mu \mathrm{m}$ above the focal plane will be larger than the opening itself. In fact, this constraint limits the numerical aperture of the objective lens to approximately 0.8 .

The simulations were performed by rigorously solving Maxwell's equations for the focused field in and near the $\mu$-SIL, since vectorial diffraction effects cannot be neglected when dealing with small objects interacting with light. To mimic the experimental conditions, an azimuthally polarized laser light, at wavelength $642 \mathrm{~nm}$, is focused by a NA = 0.8 objective (from the top to the bottom) onto the planar bottom surface of the $\mu$-SIL, as shown in the bottom right in figure 1. The geometrical focus of the high NA lens is chosen to be at $z=0 \mathrm{~nm}$, coincident with the bottom of the $3 \mathrm{D}$ hemispherical $\mu$-SIL $(n=1.5)$. The surrounding space is filled with air $(n=1)$. An adaptive meshing technique was employed to assign a higher number of points in the central region of the computation domain, in order to ensure a good representation of the rounded $\mu$-SIL structure. Elements of order 2 were used on a hexahedral mesh with total size of $4000 \times 4000 \times 1350 \mathrm{~nm}^{3}$. This particularly large computational domain is necessary in order to completely fit the $\mu$-SIL at displaced positions. A perfect matched layer was defined in all directions to truncate the computational domain. The excitation focused spot, defined as the spot generated by the objective lens, is computed at the focal plane of that lens by solving the Debye integral. These values are Fourier transformed. Each plane wave is back propagated in the $z$-direction and for a set of $z$-planes the electric fields for these waves are calculated. The back-Fourier-transforms of these fields are used in the calculation of the right-hand side of the FEM formulation of the vector-Helmholtz equations for the optical phenomena. The simulations were performed in a cluster of four AMD Quad-Core Opteron 6176 $(2.3 \mathrm{GHz})$ with $256 \mathrm{~Gb}$ of internal memory, operating under a Linux environment. The total execution time for a single computation is about $2 \mathrm{~h}$, consuming approximately $10 \%$ of the total memory.

In figure 2, the measured and simulated immersed focal spots, normalized to unity, are mapped in a circular measuring window $2 \mu \mathrm{m}$ in diameter for the cases of the doughnut and two half-lobe spots. In both cases, the $\mu$-SIL is centred in the $z$-axis. The measured results for the doughnut spot, shown in figure 2(a), show a measured spot slightly broader than that of the simulated counterpart, mainly in the outer rim of the annular spot. This difference is most likely to be due to deviations of the shape of the SIL from the ideal half-spherical shape. For the two half-lobe spots, similar results are verified, as can be seen in figure 2(b). In particular, the measurements present a more rounded spot in comparison with that of the simulations. The broadening along the $y$-direction in the simulated spot comes from the non-vanishing $z$-component of the electric field, which is not detected by the HRIM setup. Nonetheless, a reasonably good agreement between simulation and measurement is found. 


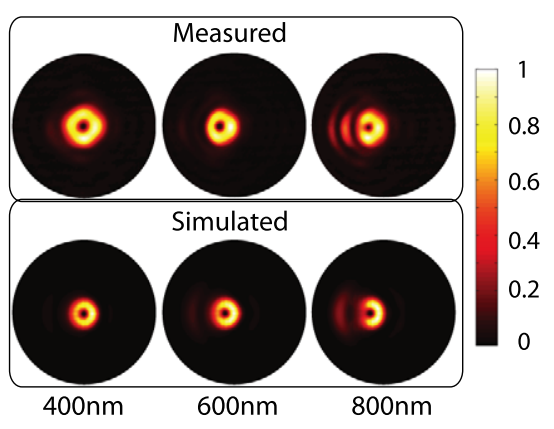

(a)

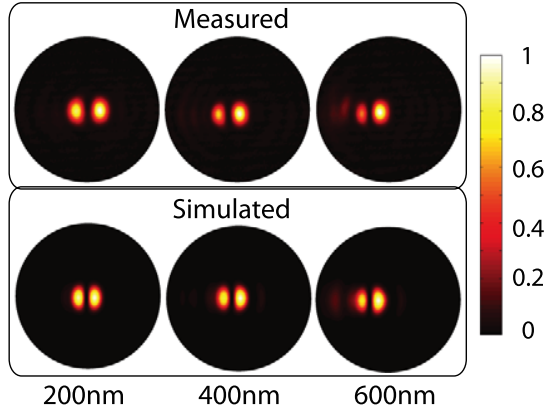

(b)

Figure 3. Measured and simulated off-axis immersed spot. (a) Doughnut-like immersed spot and (b) two half-lobe immersed spots, without the $z$-component of the electric field. The $\mu$-SIL is misaligned in the $x$-direction. The measuring circular window has a diameter of $4 \mu \mathrm{m}$. All intensities are normalized.

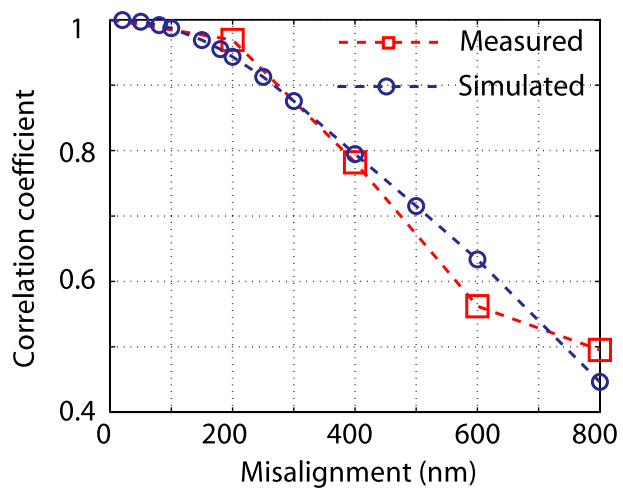

(a)

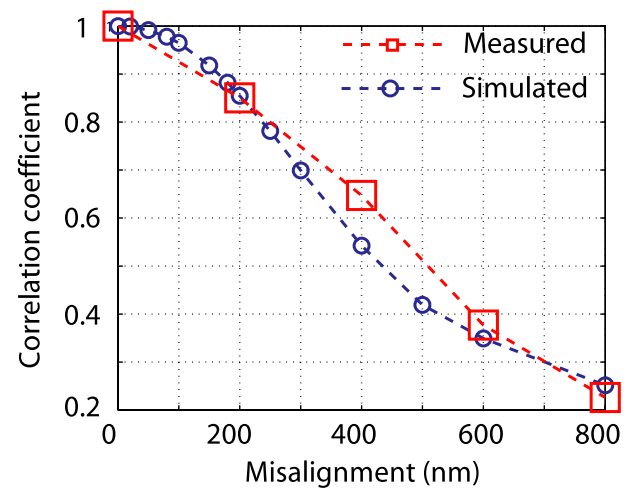

(b)

Figure 4. Measured and simulated correlation considering displacements along the $x$-direction for (a) azimuthally polarized light and (b) two half-lobe spots. A high correlation coefficient indicates that the shape of the immersed spot is preserved. The cut-off value is found to be 0.8 .

\section{On the misalignment tolerance}

A set of measurements/simulations were performed with the $\mu$-SIL misaligned with respect of the $z$-axis, by moving it in the $x$-direction, as indicated by the crosses in the central diagram on the right box of figure 1 . We initially consider the case where the doughnut spot is immersed. As seen from the results, displayed in figure 3(a), a significant distortion of the immersed doughnut spot occurs if the $\mu$-SIL is in large misalignment with respect to the $z$-axis. If the centre of the SIL deviates $800 \mathrm{~nm}$ in the $x$-direction, the doughnut shape is almost entirely lost. In practice, it would not be possible to trap a particle under this condition since a low intensity gradient occurs at one side of the spot. Similarly, a deviation of $600 \mathrm{~nm}$ still affects considerably the spot shape, though less severe than in the previous case. However, if a displacement of $400 \mathrm{~nm}$ or less is considered, the focused spot starts to resemble its initial, not-deformed, shape. It represents, nonetheless, a considerably large misalignment tolerance, considering that the SIL has only $1 \mu \mathrm{m}$ of radius. The same observations apply for the two half-lobe spots, where the effect of the misalignment of the $\mu$-SIL on the immersed spot is evidenced by a shift in the electric field energy density towards one of the lobes (see figure 3(b)). However, the results indicate a tighter margin for misalignment tolerance. It can be easily seen that a displacement of $200 \mathrm{~nm}$ is the maximum tolerable for this case.

To better quantify the tolerance error, the correlation (normalized covariance) between the misaligned and the ideal focused spots are computed. Hence, highly correlated spots indicate that the shape of the immersed spot is preserved. In figure 4 , the simulated and measured correlations, obtained for misaligned spots along the $x$-direction, are plotted as a function of the misalignment distance. The result for the doughnut spot, displayed in figure 4(a), indicates a high correlation coefficient for $200 \mathrm{~nm}$ displacement, of more than $95 \%$. At $400 \mathrm{~nm}$, the correlation drops to $80 \%$, setting the bottom acceptable limit since the immersed spot still retains a reasonably good doughnut shape. Beyond this point, the spot deviates considerably from the ideal, as discussed before. For the two half-lobe case, the correlation value corresponding to a displacement of $200 \mathrm{~nm}$ is also around 0.8 . This is the limit tolerable for this particular polarization case. From this discussion, it is noticeable that the misalignment tolerance is dependent on the polarization of the incoming focused beam that undergoes immersion. Therefore, we extend our simulation model to study the misalignment tolerance for laser beams having polarizations other than azimuthal. 


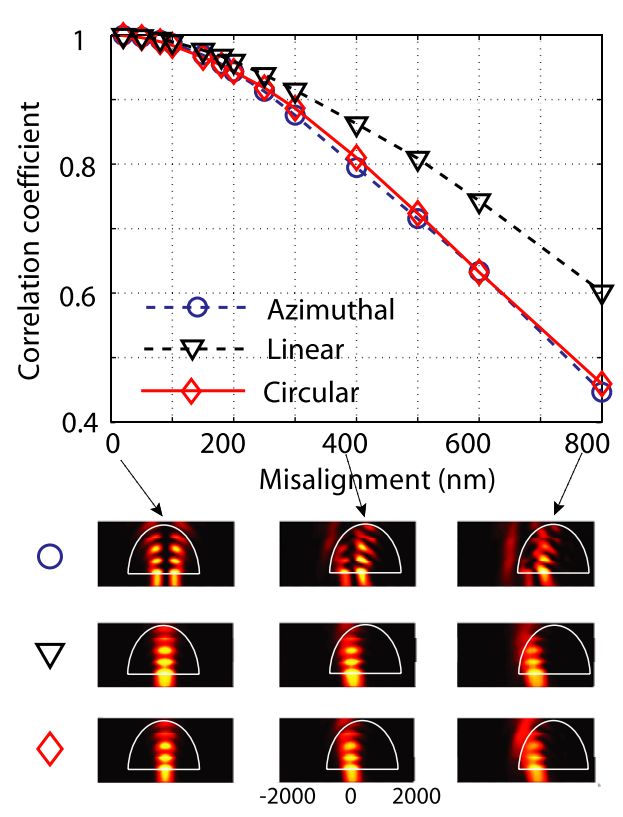

(a)

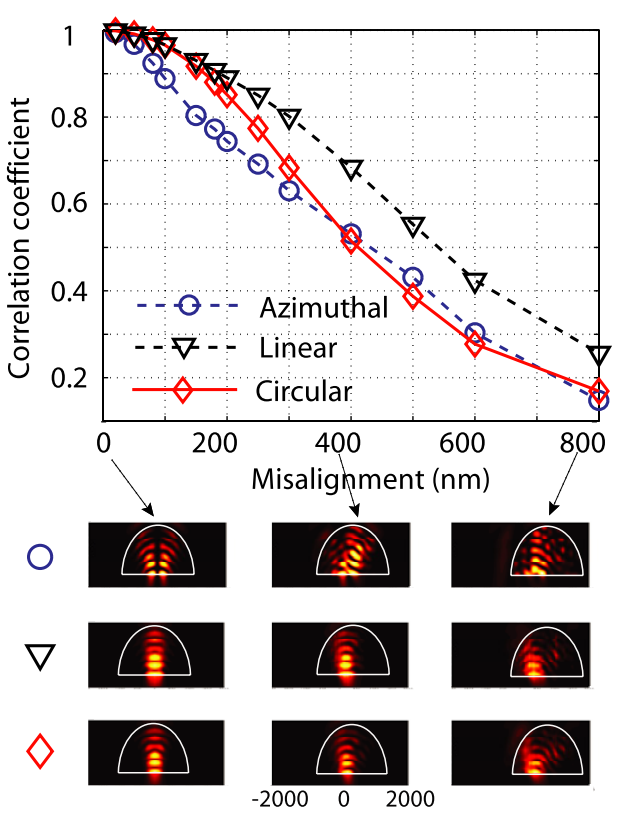

(b)

Figure 5. Simulated correlation computed for the $\mu$-SIL having refractive index of (a) $n=1.5$ and (b) $n=2$, with the corresponding intensity distributions as the light passes through the SIL. Provided that the incident focused spot falls entirely onto the $\mu$-SIL, a high correlation is assured.

\section{Other cases of interest}

In figure 5, the correlation is displayed for light beams with circular, azimuthal and linear polarizations. We initially consider the same $\mu$-SIL, that is, a $\mu$-SIL with $2 \mu \mathrm{m}$ diameter and refractive index of 1.5. The results, displayed in figure 5(a), indicate an even higher misalignment tolerance for the circular and linear polarizations. For the circularly polarized immersed spot, a misalignment slightly larger than $400 \mathrm{~nm}$ shows $80 \%$ of correlation with respect of the on-axis spot. The linearly polarized immersed spot, in a similar way, still retains a reasonably good shape even if the $\mu$-SIL is misaligned by $500 \mathrm{~nm}$ with respect to the z-axis. In fact, the condition to achieve such a high correlation can be inferred from the total electric field distribution computed along the $x z$ plane, displayed on the bottom of that figure; provided that the incident focused spot is entirely incident within the $\mu$-SIL, a high correlation is obtained. This observation sets a practical guide for designing and evaluating optical immersion systems operating with objectives with different numerical apertures and/or different $\mu$-SIL size. These observations, however, depend on the refractive index of the material of which the $\mu$-SIL is made. In figure 5(b), we repeat the simulations after replacing the initial refractive index, $n=1.5$, by $n=2$. SiN is an example of a high refractive index material that has been used for manufacturing $\mu$-SILs. As can be seen, the correlation drops considerably under this circumstance. In fact, the misalignment tolerance drops to about half of what was previously found. The total electric field distribution, displayed on the bottom of the figure, indicates that the stronger interference effect inside the $\mu$-SIL is detrimental to the final immersed spot.

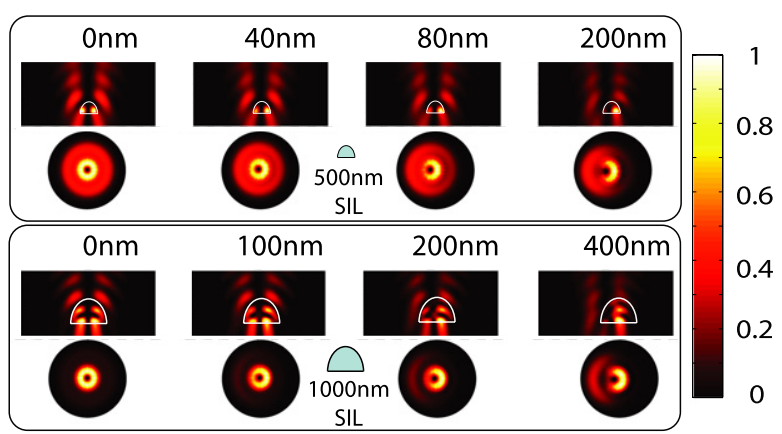

Figure 6. Simulated intensity distribution of the immersed spots generated by different types of $\mu$-SIL. Top: $\mu$-SIL with diameter of $500 \mathrm{~nm}$. Bottom: $\mu$-SIL with diameter of $1000 \mathrm{~nm}$. The misalignment tolerance is highly dependent on the size of the $\mu$-SIL.

As a last example, we consider the misalignment tolerance of $\mu$-SILs with diameter smaller than $2 \mu \mathrm{m}$, namely, $1 \mu \mathrm{m}$ and $0.5 \mu \mathrm{m}$. The refractive index is kept as $n=1.5$, the NA of the focusing lens is 0.8 and the polarization of the illumination beam is set to azimuthal. We plot the simulation results in figure 6 . For $\mu$-SIL with diameter of $500 \mathrm{~nm}$, the shape of the doughnut spot is entirely lost after a displacement of $80 \mathrm{~nm}$, which represents $1 / 6$ of the lens diameter. The $\mu$-SIL with diameter of $1000 \mathrm{~nm}$ showed a reasonably good immersed spot even misaligned by $100 \mathrm{~nm}$. At $200 \mathrm{~nm}$, however, the immersed spot no longer shows a good doughnut shape.

\section{Conclusions}

We experimentally and theoretically investigated the alignment error tolerance of a $2 \mu \mathrm{m}$-SIL immersing beam with two 
types of polarization. It was found that a large misalignment with respect to the $z$-axis of an objective lens is tolerable. In fact, such misalignment can be as large as $400 \mathrm{~nm}$, i.e. one fifth of the lens diameter for the azimuthally polarized beam and $200 \mathrm{~nm}$ for the two side-lobes spot. The numerical study was then extended to account for different polarizations. The $\mu$-SIL can still generate a good immersed spot for a circularly polarized beam even if displaced by approximately $420 \mathrm{~nm}$. The tolerance is even higher for linearly polarized beams, reaching up to one-fourth of the lens diameter. Furthermore, we discussed the basic condition for not compromising the immersed spot integrity. It suffices that the incident spot falls entirely inside the SIL. Moreover, we discussed the misalignment tolerance of different types of $\mu$-SIL. It was found that the misalignment tolerance is reduced for $\mu$-SIL made of materials with higher refractive index. At last, we numerically investigated the misalignment tolerance of smaller $\mu$-SILs. It was shown that smaller $\mu$-SILs are more sensitive to alignment errors. To conclude, the present study contributes to a better understanding of the misalignment tolerance of a micro-sized solid immersion lens. The exceptionally large misalignment tolerance makes the $\mu$-SIL a promising candidate to improve not only trapping efficiency, but also many advanced optical systems.

\section{Acknowledgment}

This work is financially supported by the FP7 European Project IST-SURPASS, Project Number 224226.

\section{References}

[1] Kim M-S, Scharf T, Haq M T, Nakagawa W and Herzig H P 2011 Subwavelength-size solid immersion lens Opt. Lett. 36 3930-2

[2] Fletcher D A, Crozier K B, Quate C F, Kino G S, Goodson K E, Simanovskii D and Palanker D V 2000 Near-field infrared imaging with a microfabricated solid immersion lens Appl. Phys. Lett. 772109

[3] Brun M, Richard M and Nicoletti S 2009 Integrated micro solid immersion lens for near field optical data storage Int. Symp. on Optical Memory (ISOM09), Mo-E-04

[4] Knieling T, Shafi M, Lang W and Benecke W 2012 Microlens array production in a microtechnological dry etch and reflow process for display applications J. Eur. Opt. Soc. Rapid Publ. 712007
[5] Mason D R, Jouravlev M V and Kim K S 2010 Enhanced resolution beyond the Abbe diffraction limit with wavelength-scale solid immersion lenses Opt. Lett. 35 2007-9

[6] Mansfield S M and Kino G S 1990 Solid immersion microscope Appl. Phys. Lett. 54 2615-6

[7] Ghislaina L P, Elings V B, Crozier K B, Manalis S R, Minne S C, Wilder K, Kino G S and Quateb C F 1999 Near-field photolithography with a solid immersion lens Appl. Phys. Lett. 74 501-3

[8] Mansfield S M, Studenmund W R, Kino G S and Osato K 1993 High-numerical-aperture lens system for optical storage Opt. Lett. 18 305-7

[9] Lerman G M, Israel A and Lewis A 2006 Applying solid immersion near-field optics to Raman analysis of strained silicon thin films Appl. Phys. Lett. 89223122

[10] Koyama K, Yoshita M, Baba M, Suemoto T and Akiyama H 1999 High collection efficiency in fluorescence microscopy with a solid immersion lens Appl. Phys. Lett. 75 1667-9

[11] Dienerowitz M, Mazilu M and Dholakia K 2008 Optical manipulation of nanoparticles: a review J. Nanophoton. 2021875

[12] Zhan Q 2009 Cylindrical vector beams: from mathematical concepts to applications Adv. Opt. Photon. 1 1-57

[13] Chu S 1998 The manipulation of neutral particles Rev. Mod. Phys. 70 685-706

[14] Hell S W and Wichmann J 1994 Breaking the diffraction resolution limit by stimulated emission: stimulated-emission-depletion fluorescence microscopy Opt. Lett. 19 780-2

[15] Škereň M, Svoboda J and Fiala P 2012 Advanced matrix laser lithography for fabrication of photonic micro-structures J. Eur. Opt. Soc. Rapid Publ. 712043

[16] Kim M-S, Scharf T and Herzig H P 2012 Vortex beam lithography Paper under peer-review

[17] Brun M, Mimoumi S, Nicoletti S, Poupinet P and Moriceau H 2010 Solid immersion lens and related method for making same US Patent Specification 2010/0200770 A1

[18] Kim M-S, Scharf T and Herzig H P 2010 Small size microlenes characterization by multiwavelength high resolution interference microscopy $O p t$. Express 18 14319-29

[19] Wachters A J and Urbach H P 2008 Technical Note Phillips Research Europe PR-TN 00042

[20] Wei X, Wachters A J and Urbach H P 2007 Finite-element model for three-dimensional optical scattering problems J. Opt. Soc. Am. A 24866

[21] Assafrao A C, Wachters A J H, Verheijen M, Nugrowati A M, Pereira S F, Urbach H P, Armand M-F and Olivier S 2012 Direct measurement of the near-field super resolved focused spot in InSb Opt. Express 20 10426-37 